\title{
¿CÓMO DESCRIBIR EL PROCESO DE ESCRITURA EN LOS AUTÓGRAFOS DE CALDERÓN? UNA TAXONOMÍA DE LAS CORRECCIONES ${ }^{1}$
}

\author{
Simon Kroll \\ Universität Wien \\ Institut für Romanistik \\ Universitätscampus AAKH, Hof 8 \\ Spitalgasse 2 \\ A-1090 Wien. Austria \\ simon.kroll@univie.ac.at
}

\section{INTRODUCCIÓN}

Estudiar autógrafos es un trabajo bastante paradójico. En el ámbito de la Filología parece que no hay nada tan dinámico y nada tan estático como un autógrafo. Quiero decir con ello que en ningún testimonio de la literatura estamos tan cerca de la inventio, dispositio y elocutio del autor como cuando leemos autógrafos. No obstante, fenomenológicamente dicho, los autógrafos aparecen como productos estáticos. Se ve un folio y sobre el folio letras y palabras, pero lo que a primera vista no se ve es el tema de enfoque de este tomo: el proceso de escritura.

Ahora bien, ¿cómo podríamos describir este proceso? Entre otras cosas se puede observar en las correcciones que realiza el propio poeta.

${ }^{1}$ Este trabajo forma parte del proyecto «Secrets and Secrecy in Calderón’s Comedies and in Spanish Golden Age Culture; including a Critical Edition of El secreto a voces» financiado por el Austrian Science Fund (P 24903-G23) y el Anniversary Fund del Oesterreichische Nationalbank (14725). Por sus correcciones y sugerencias le agradezco su amable ayuda a Fernando Rodríguez-Gallego. 
Veamos un ejemplo del autógrafo de El agua mansa. Primero cito el pasaje como ha sido editado por Ignacio Arellano y Víctor García Ruiz:

D. FÉLIX

El cielo

os guarde, y no importa nada,

que entre amigos un disgusto,

y de tan poca importancia,

nosotros le compondremos, y adiós.

Vase

D. AlONSO

Mari Nuño, baja ${ }^{2}$

El agua mansa es una de las obras calderonianas de la que disponemos de un autógrafo. Contemplando el pasaje correspondiente en él, parece que Calderón escribió primero:

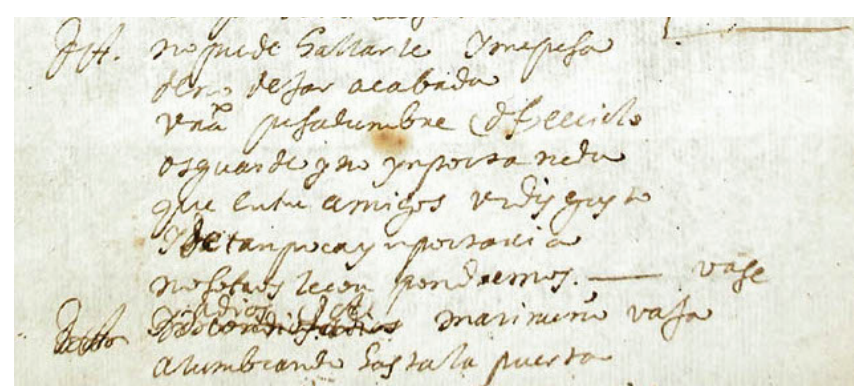

Ilustración 1: El agua mansa, Ms,VIT-197, Biblioteca de l'Institut del Teatre de la Diputació de Barcelona, fol. 48v

D. FELIX

Don Alonso
El cielo

os guarde, y no ynporta nada que entre amigos vn disgusto, y de tan poca ynportancia, nosotros le compondremos. - Vase Yd con dios. Adiós. (fol. 48v) ${ }^{3}$

${ }^{2}$ Calderón, El agua mansa/ Guárdate del agua mansa, p. 342, vv. 2596b-2601.

3 Se puede consultar el autógrafo cervantesvirtual.com: <http://www. cervantesvirtual.com/obra-visor/el-agua-mansa-manuscrito-comedia-en-tres-actos--0/ html/0217e43c-82b2-11df-acc7-002185ce6064_97.html>. 
¿Por qué opino que Calderón escribió este verso un poco raro de «Id con dios. Adiós» pronunciado por un personaje, cuando en Calderón sería mucho más corriente poner este verso en boca de dos personajes? Solo recuerdo los ejemplos de versos compartidos al estilo de "Don Alonso. Id con Dios. Don Félix. Adiós». En el autógrafo se puede observar bien cómo Calderón colocó la acotación referente a don Félix —el Vase - tras el verso «nosotros le compondremos». Es decir, que ya se habría ido en el verso siguiente. Esto podría ser un pequeño despiste, pero hay otro indicio para mi hipótesis. La indicación de locutor en el supuesto verso compartido por don Alonso y don Félix se realizó por encima de la «s» de «dios» en «Id con dios». Apenas se ve, pero el punto después de la «s» revela, a mi modo de ver, que se ha añadido en este caso una indicación de locutor. El hecho de que se añada la «f» de Félix posteriormente me parece que queda comprobado por haberse escrito sobre la «»» de «dios». Si el análisis acierta, Calderón intenta corregir el verso «id con dios adiós» de Félix en «Don Alonso. Id con dios. Don Félix. Adiós». A esta altura del verso tiene pocas opciones para terminarlo. De las ocho sílabas le quedan solo tres, de las cuales dos ya están reservadas para las vocales á-a por las exigencias de la rima. Es decir, que tiene solo una sílaba a su libre disposición. Si atendemos al texto que sigue podría especularse que una posible solución de terminar este verso habría sido: «Don Alonso. Id con dios. Don Félix. Adiós. Don Alonso. Tú baja». Claro está, esto es pura especulación. Pero también está claro que "Mari Nuño", con sus cuatro sílabas, no cabría de ninguna manera en la medida del verso. Lo que sabemos con seguridad es que Calderón no encontró solución satisfactoria a estas cinco sílabas. Tachó por consiguiente todas las sílabas y las indicaciones de locutor y añadió otro «Adiós» entre los versos, este todavía en boca de don Félix. Ahora, teniendo cuatro sílabas a su libre disposición (más las dos de la rima), termina el verso en «Don Alonso. Mari Nuño baja». En esta corrección podemos observar cómo Calderón construye sus versos y cómo trabaja con el cómputo de la medida del verso. En especial es interesante que por lo visto prefiere soluciones en las que puede incorporar los nombres propios.

En otro ejemplo, extraído de El secreto a voces, ocurre algo parecido. $\mathrm{El}$ atajo en el folio $16 \mathrm{v}$ provoca correcciones en el verso adyacente, que en una primera versión decía «desvelar, y así de ti»". Calderón tacha «desvelar», y aprovecha las sílabas liberadas para incorporar el nombre de Laura.

${ }^{4}$ Del autógrafo de El secreto a voces se trata con más detalle en Kroll, 2014. 


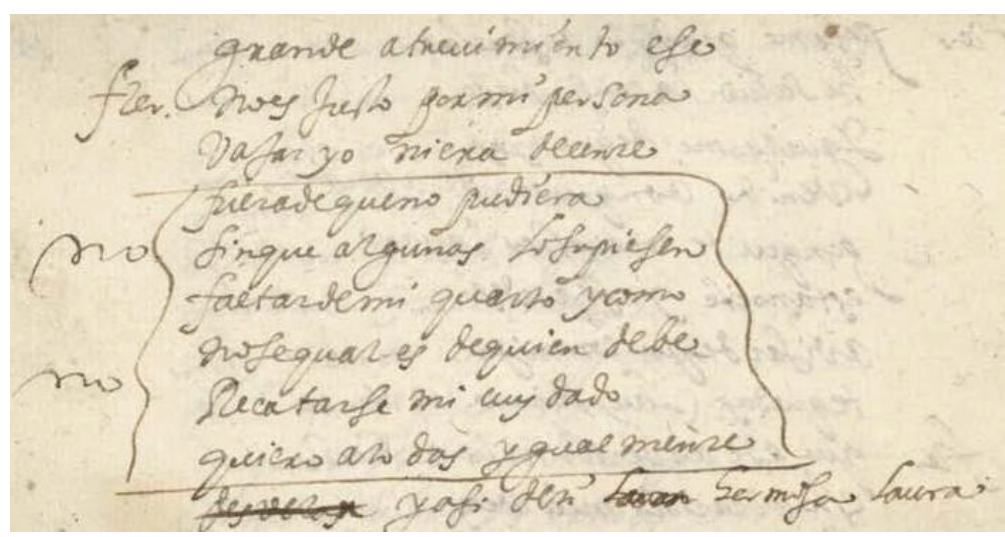

Ilustración 2: El secreto a voces, Ms, Res. 117, BNE, fol. 16v

Ahora bien, si dejamos la reconstrucción de una corrección tal cual, por muy minuciosa que sea, echaremos en falta una clasificación. Ya Ruano de la Haza había propuesto diferenciar los procesos de reescritura en refundición, reelaboración, reconstrucción, adaptación y reutilización ${ }^{5}$. Para clasificar las correcciones propiamente dichas nos interesarían sobre todo los términos «reelaboración» y «adaptación». Cabe recordar que reelaboración es, según Ruano de la Haza, el proceso mediante el que un escritor «pule, perfecciona, afina y modifica un texto teatral para crear una nueva versión, y puede ser llevada a cabo por el propio dramaturgo, como hace, por ejemplo, Calderón con su La vida es sueño» ${ }^{6}$. Adaptación sería «la práctica de adecuar un texto teatral, eliminando personajes o escenas que se consideran superfluos, o introduciendo nuevos pasajes y personajes, para producir una versión representable en un lugar escénico específico o por una compañía determinada» ${ }^{7}$. Adaptación y reelaboración pueden realizarse sobre distintos niveles del texto, pueden atañer a los personajes, al texto de un personaje, a ciertos pasajes o incluso a ciertas palabras o rimas.

Para diferenciar los distintos tipos de corrección propongo clasificarlas según las distintas fases de composición de un discurso que establecía la Retórica clásica. En especial nos interesarán la inventio, la dispositio y la elocutio. Aunque por encima de todo sería quizá útil introducir un as-

\footnotetext{
${ }^{5}$ Ruano de la Haza, 1998.

${ }^{6}$ Ruano de la Haza, 1998, p. 35.

${ }^{7}$ Ruano de la Haza, 1998, p. 35.
} 
pecto temporal: el momento de la corrección. ¿Se realiza in itinere o en un momento posterior? El ejemplo comentado de El agua mansa parece corresponderse con el primer tipo de correcciones, mientras que el de El secreto a voces es bastante probablemente un ejemplo de una corrección en un momento posterior. Después habría que analizar sobre qué fase de producción textual se lleva a cabo la corrección.

\section{LA INVENTIO}

En lo que respecta a la inventio pueden darse intervenciones sobre el tema, el título, los personajes, el lugar o el tiempo. Obviamente no cada personaje añadido o eliminado ocasiona una intervención sobre la inventio. La corrección tendría que cambiar una idea axial de la trama. Añadir un ángel para tener dos en vez de uno, no entraría debajo de esta categoría ${ }^{8}$.

Me pregunto, en cambio, si las intervenciones sobre las dramatis personae y el título del auto El divino cazador se podrían clasificar como correcciones de la inventio, pues, como se puede apreciar en la ilustración 3, Calderón tacha el primer título, que decía La fiera de los montes, y añade el nuevo de El divino cazador por encima del tachado.

Además, se elimina el personaje del Lucero. Las demás tachaduras son intervenciones menores, pues el Rey, por ejemplo, a pesar de estar tachado, aparece luego en el texto. La eliminación del Lucero tiene que ser posterior a la escritura del texto, ya que en este aparecen versos en un primer momento previstos para el Lucero (fol. 10v) ${ }^{9}$. En los pocos versos que iba a decir se ha corregido la indicación de locutor de el Lucero en una Voz (fols. 10v, 11r) o incluso se pasan a la boca del Príncipe, trasunto de Cristo (fols. $11 \mathrm{v}$ y $14 \mathrm{v}$ ). Se trata de intervenciones de no poco peso, porque ya la corrección del título cambia el enfoque del auto. La fiera del monte es en este caso la Culpa, mientras que El divino cazador es la figura alegórica de Cristo.

${ }^{8}$ Así parece ocurrir en el autógrafo de La humildad coronada. Compárese el fol. 1v del autógrafo, accesible en el portal cervantesvirtual.com: $<$ http://www.cervantesvirtual. com/obra-visor/la-humildad-coronada-manuscrito-auto-sacramental-para-la-ciudad-detoledo-en-las-fiestas-del-santisimo-sacramento-ano-de-1644-inc-arboles-plantas-y-floresexp-lograr-la-corona-y-cenir-el-laurel--0/html/> [27/01/2015]. Hay una edición crítica del auto preparada por Ignacio Arellano: Calderón de la Barca, La humildad coronada, p. 59.

${ }^{9} \mathrm{El}$ autógrafo de El divino cazador está accesible en el portal cervantesvirtual.com: $<$ http://www.cervantesvirtual.com/obra/el-divino-cazador-manuscrito-auto-sacramental-inc-verde-apacible-esfera-exp-todos-a-un-punto-me-faltan--0/> [27/01/2015]. 
Cambiar el título tal y como lo hace Calderón lleva el enfoque de la Culpa a su redención en Jesucristo, lo que es un cambio considerable.

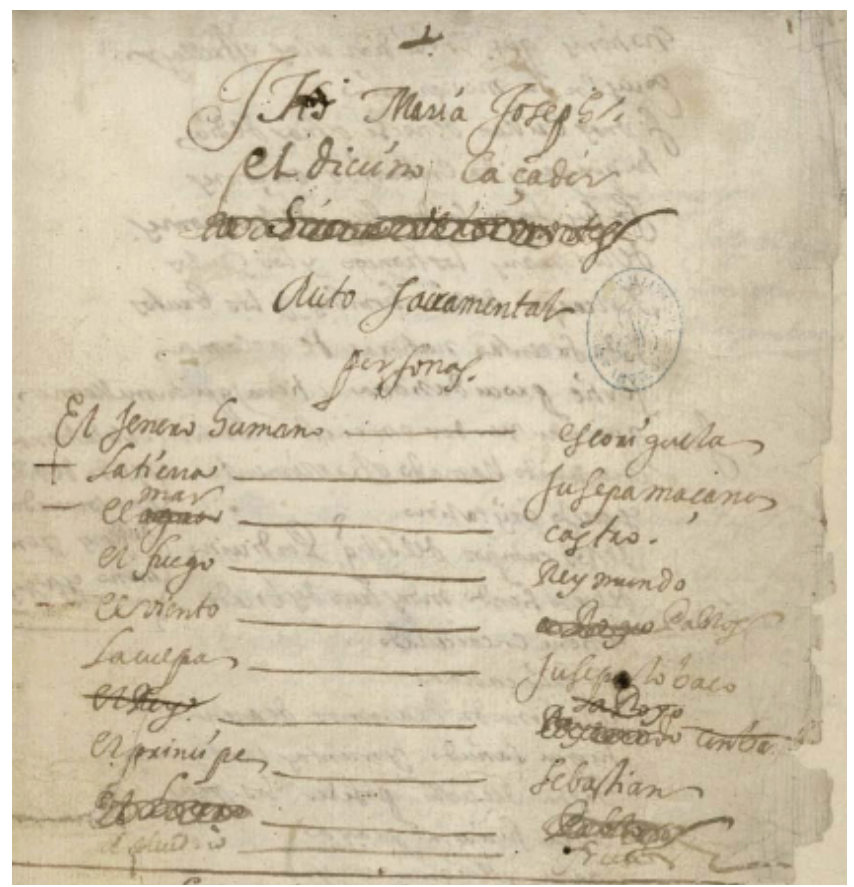

Ilustración 3: El divino cazador, Ms, Res. 268, BNE, fol. 1r

La eliminación del Lucero solo afecta a muy pocos versos, pero supone, de todas maneras, la desaparición de una figura alegórica. El análisis del autógrafo de El divino cazador deja ver que el caso es bastante complicado. Si nos fijamos en los versos que en una primera versión pronunciaba el Lucero en los folios 10v y 11r, vemos en seguida que son versos poco típicos de un Lucero, pues dice en el folio 10v: «Danos señor a tu hijo / envíanos la salud» ${ }^{10}$, y en el folio 11r: «Todos en Cristo sanaron / del contagio de la muerte» ${ }^{11}$. Estos versos atípicos de un demonio invitan al menos a pensar que la corrección del locutor en el texto se realizó sobre la marcha. Más evidente se hace esta hipótesis en el folio $14 \mathrm{v}$. En este se han tachado algunos versos del Lucero y también

${ }^{10}$ Calderón, El divino cazador, fol. 10v, vv. 599-600.

${ }^{11}$ Calderón, El divino cazador, fol. 11r, vv. 604-605. 
algunos del Príncipe. Es bastante dificil descifrar lo tachado, pero parece que los últimos dos versos tachados dicen: «Príncipe.Yo no / porque aunque a matarla venga / he de vencerla muriendo» ${ }^{12}$. Ya que estos versos tachados no enlazan en absoluto con el verso no tachado que sigue, "no he de vencerla naciendo", podemos decir que estas correcciones debieron de realizarse sobre la marcha y no en un momento posterior. Lo que sigue en la columna y los versos en el margen corresponden, por tanto, a una versión corregida, como se puede apreciar en la ilustración 4.

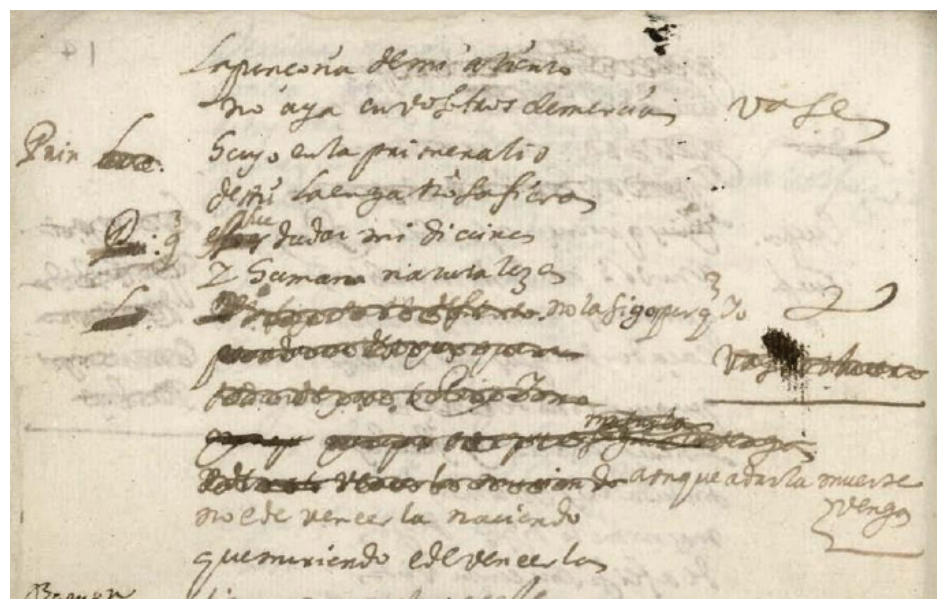

Ilustración 4: El divino cazador, Ms, Res. 268, BNE, fol. 14v

Calderón tachó, por lo visto, al menos algunos de los versos del Lucero durante la preparación del autógrafo que se ha conservado y volvió después al reparto para eliminar el personaje definitivamente. En resumidas cuentas, se puede decir que las intervenciones sobre el título y las dramatis personae justifican que se hable al menos de correcciones leves sobre el nivel de la inventio aunque la trama del auto no se vea alterada en su esencia por dichas correcciones.

\footnotetext{
${ }^{12}$ He aquí la transcripción diplomática:

$<$-Pr. Yo no

$<$-porque $>$ porque aunque a $<-$ ? $>$ matarla venga

e de vencerla muriendo $>$

no e de vencerla naciendo
} 


\section{LA DISPOSITIO}

En el nivel de la dispositio podemos diferenciar entre la eliminación, la permutación y el añadido de escenas, pasajes o pasos. El caso del folio 11r del autógrafo de La desdicha de la voz $z^{13}$ se puede entender como una permutación en el nivel de la dispositio, aunque también esté acompañado por importantes reelaboraciones en el nivel de la elocutio. Veamos el caso en detalle. El texto del folio 11r se ha tachado enteramente y, según el análisis de Mason, debe corresponderse con una versión previa al estado textual de las demás partes del autógrafo. Mason opina que los folios 7 a 10 del autógrafo conservado ofrecen una segunda versión de estos pasajes. El folio 11r sería el último resto de la versión previa. «Thus it seems that folios 7 to 10 inclusive of the present manuscript must at some stage have replaced an earlier version, the only surviving trace of which is the wholly deleted folio $11 \mathrm{r}{ }^{14}$.

Es decir, que Calderón debió de descartar los pasajes de los folios 7 a 11r en su versión previa, sustituir los folios y pasar la versión corregida a limpio sobre los folios nuevos. El último resto que quedó de la versión ante correctionem debe de ser el folio 11r. Parece, además, que Calderón añadió la versión en un momento posterior, ya que al final del folio $10 \mathrm{v}$ empieza a escribir en dos columnas para que el último verso de este folio enlace bien con el primero del folio 11v. Lo que importa de esta reconstrucción es que al parecer se retocó considerablemente un pasaje de varios folios de extensión.

Aclarados estos detalles, veamos la permutación sobre el nivel de la dispositio. En la versión ante correctionem Calderón hizo salir a Leonor mucho más tarde, pues, como se aprecia en el folio 11r, solo iba a salir a estas alturas del texto. La versión corregida prevé la salida de Leonor ya en el folio 8v, por lo que se realiza mucho más temprano. Lo mismo subraya Mason en su análisis del autógrafo: "The dramatic effect of this revision must have been considerable, for in the new version, the arrival of Doña Leonor is placed much earlier than before [...]. She comes not alone, but accompanied by her father, and they discuss the forthcoming trip to Seville» ${ }^{15}$.

${ }^{13}$ El autógrafo de La desdicha de la voz está accesible en el portal cervantesvirtual. com: <http://www.cervantesvirtual.com/obra/la-desdicha-de-la-voz-manuscrito-comedia-inc-amiga-mia-ya-sabes-exp-que-perdoneis-nuestras-faltas--0/> [27/01/2015].

${ }^{14}$ Mason, 2003, p. 6.

${ }^{15}$ Mason, 2003, p. 6. 
Como se ha movido una salida de un personaje de manera considerable, podemos llamar esta corrección una permutación de una escena sobre el plan de la dipositio.

\section{LA ELOCUTIO}

En lo que se refiere a la elocutio pueden darse correcciones sobre el metro y el estilo.

A modo de ejemplo me gustaría presentar una corrección estilística. El siguiente pasaje tomado del autógrafo de El secreto a voces es sugerente, porque revela un aspecto interesante del trabajo de Calderón sobre su propio texto. Federico y Fabio hablan de una carta que Flérida le había dado a su secretario Federico, y dice este:

$\begin{array}{ll}\text { Federico } & \begin{array}{l}\text { Aun el sobrescrito de ella } \\ \text { no me he atrevido a leer. }\end{array} \\ \text { Fabio } & \begin{array}{l}\text { Léele, a ver si contradice } \\ \text { a lo que primero fue }\end{array}\end{array}$

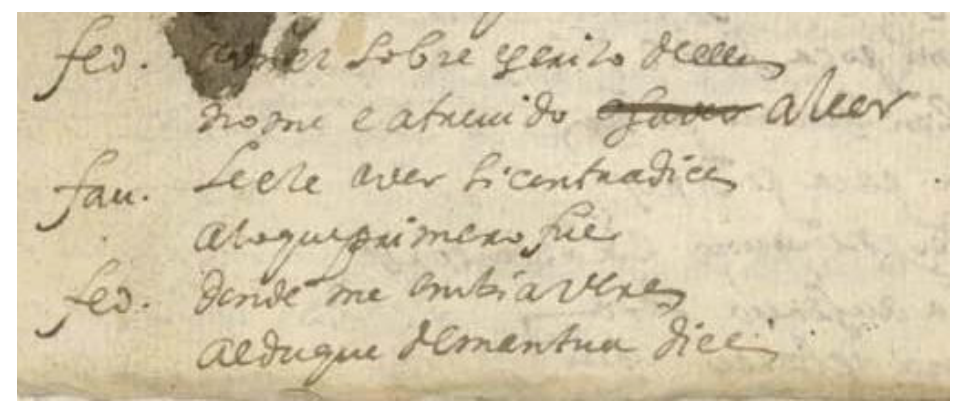

Ilustración 5: El secreto a voces, Ms, Res. 117, BNE, fol. 13v

Como bien se puede apreciar en el autógrafo (ilustración 5), Calderón escribió primero: "Aun el sobrescrito de ella / no me he atrevido a saber». No hay ninguna ocurrencia de la palabra «saber» sobre el folio, por lo que podemos descartar que se trata de un simple salto de ojo que Calderón luego corrigiese en «a leen». Fueran los que fueran sus motivos para escribir primero «a saber», la corrección en «a leer» nos da indicios importantes sobre el trabajo estilístico-retórico de Calderón, ya que revela cómo busca figuras retóricas de

${ }^{16}$ Calderón, El secreto a voces, vv. 792-795. 
repetición, pues aquí se forma en la versión corregida una anadiplosis entre «leer» y el «léele» del verso siguiente. Calderón afina su texto.

\section{Conclusiones}

Calderón no cesa de retocar sus textos. MarcVitse afirma que Calderón es "por antonomasia, el dramaturgo áureo de la reescritura» ${ }^{17}$. La crítica textual detecta cada vez más textos con varias versiones ${ }^{18}$ y ha producido una vasta terminología sobre el asunto, como la ya mencionada distinción establecida por Ruano de la Haza entre diferentes tipos de reescritura, o la que distingue entre textos con variantes y variantes de textos ${ }^{19}$. A pesar de que contamos con una nomenclatura bien elaborada para diferenciar los distintos tipos de reescritura, podría ser útil introducir una taxonomía para el microanálisis de cada corrección que introduce el poeta sobre su texto. Para estos fines he elaborado la taxonomía de correcciones que vengo describiendo y que podría resumirse en la siguiente tabla:

\begin{tabular}{|l|l|l|}
\hline \multicolumn{3}{|c|}{ Momento de la corrección } \\
\hline Inventio & Dispositio & Elocutio \\
\hline Tema & Eliminación & Metro \\
\hline Título & Permutación & Estilo \\
\hline $\begin{array}{l}\text { Personajes } \\
\text { Espacio dramático } \\
\text { Tiempo dramático }\end{array}$ & Añadidura \\
\hline \multicolumn{2}{|c|}{ Correcciones de lapsus } \\
\hline
\end{tabular}

${ }^{17}$ Vitse, 1998 , p. 6.

18 El libro más importante al respecto es sin duda el de Ruano de la Haza (1992) sobre la primera versión de La vida es sueño. Pueden verse además recientes ediciones de obras como El astrólogo fingido, El mayor monstruo del mundo, Judas Macabeo, El agua mansa/ Guárdate del agua mansa o La devoción de la cruz de las que existen varias versiones, según las opiniones de los respectivos editores. Un estado de la cuestión fue elaborado por Rodríguez-Gallego, 2010.

${ }^{19}$ Arellano, 2007, p. 15. De acuerdo con Arellano, en el caso de textos con variantes las diferencias que muestran dos testimonios de un texto son menores, mientras que en el de variantes de textos son de tal envergadura que tenemos que considerar la existencia de dos versiones de una comedia. 
¿Para qué propongo clasificar las correcciones como lo acabo de presentar? Espero que con este sistema de lectura se pueda describir mejor el proceso de escritura del poeta y el momento de este proceso que representan los autógrafos. Claro está, cuando un manuscrito tiene muchas correcciones es indicio de que se trata de un borrador; y si un manuscrito tiene pocas es probable que se trate de una copia en limpio. Distinguir entre borradores y copias en limpio es quizá poco operativo. Por un lado, es una cuestión muy controvertida si disponemos de borradores calderonianos ${ }^{20}$. Por otro lado, es posible que una copia en limpio se volviese a trabajar hasta tal grado que llegó a aparecer como un borrador. En algunos casos, pienso en el autógrafo de La desdicha de la voz, se han retocado solo partes del texto, de manera que la mayoría del autógrafo tiene todo el aspecto de una copia en limpio, mientras que en algunos folios se ha trabajado muy intensamente. Calderón podía volver a escribir en cualquier momento.

Con todo, creo que con el sistema presentado se puede afinar esta interpretación y determinar mejor cuál es el estado de creación reflejado por un autógrafo y por los folios que lo componen. Si, por ejemplo, se detectan importantes intervenciones sobre la inventio o también la dispositio, podemos decir que se trata de un fuerte indicio de que la pieza se ha adaptado (siempre siguiendo la definición de Ruano de la Haza) a algún lugar o compañía en concreto. Las correcciones sobre el reparto en El divino cazador parecen indicar eso mismo. El actor/músico que interpreta al Lucero, Pablo Rodríguez, aparece por primera vez en 1642 — año de estreno del auto según M. Greer ${ }^{21}$ — en la compañía de Antonio de Prado. Tenemos noticia de él en 1643; en 1644 no sabemos nada de él, pero ya en 1645 forma parte de la compañía de Luis López ${ }^{22}$. Pablo Rodríguez no desaparece del reparto, porque tras la eliminación de su papel pasa a interpretar al Viento, mientras que el actor del Viento pasa a interpretar al Rey, personaje que quizá en adaptaciones posteriores también se haya tachado.Vemos, pues, cómo Calderón va ajustando el reparto para la compañía de Antonio de Prado. Como he podido mostrar que las intervenciones sobre los versos del Lucero se realizaron sobre la marcha, podemos formular la hipótesis de que estas correcciones se deben quizá al contacto entre compañía, censura y poeta, y

\footnotetext{
${ }^{20}$ Ver el artículo de Erik Coenen en este mismo tomo.

${ }^{21}$ Greer, 1997, p. 223.

22 DICAT, entrada Pablo Rodríguez.
} 
que Calderón efectivamente va adaptando su auto para la compañía de Antonio de Prado ${ }^{23}$.

Cuando las correcciones sobre la elocutio son muy abundantes es probablemente justificado hablar de reelaboración, como lo hace Ruano de la Haza. Esta reelaboración puede crear dos versiones de una obra. Pero parece que sobre todo las correcciones en la elocutio dan fe del Calderón que no cesa de pulir, perfeccionar, afinar y mejorar. En fin dan fe de Calderón en su laboratorio.

${ }^{23}$ Ver también Kroll, 2015 (en prensa). 


\section{BiBLIOGRAFÍA}

Arellano, Ignacio, Editar a Calderón, Madrid / Frankfurt, Iberoamericana / Vervuert, 2007.

Calderón de la Barca, Pedro, El agua mansa, autógrafo, Biblioteca del Instituto de Teatro, Barcelona, VIT. 197, <http://www.cervantesvirtual.com/obra/ el-agua-mansa-manuscrito-comedia-en-tres-actos--0/> [04/07/2014].

Calderón de la BarCa, Pedro, El agua mansa/Guárdate del agua mansa, ed. Ignacio Arellano y Víctor García Ruiz, Kassel, Reichenberger, 1989.

Calderón de la BArca, Pedro, El astrólogo fingido, ed. Fernando RodríguezGallego, Madrid / Frankfurt, Iberoamericana / Vervuert, 2011.

Calderón de la BArCa, Pedro, El divino cazador, autógrafo, BNE, Res. 268, $<$ http://www.cervantesvirtual.com/obra/el-divino-cazador-manuscritoauto-sacramental-inc-verde-apacible-esfera-exp-todos-a-un-punto-mefaltan--0/> [04/07/2014].

Calderón de la Barca, Pedro, El divino cazador, ed. Hans Flasche y Manuel Sánchez Mariana, Madrid, Ministerio de Cultura, 1981.

Calderón de la Barca, Pedro, El mayor monstruo del mundo/El mayor monstruo, los celos, ed. María J. Caamaño Rojo, Madrid / Frankfurt, Iberoamericana / Vervuert, en prensa.

Calderón de la Barca, Pedro, El secreto a voces, autógrafo, BNE, Res. 117, $<$ http://www.cervantesvirtual.com/obra/el-secreto-a-voces-manuscritocomedia-inc-razon-tienes-corazon-exp-humildes-a-vuestras-plantas--0/> [04/07/2014].

Calderón de la BArca, Pedro, El secreto a voces, ed. Wolfram Aichinger y Simon Kroll en colaboración con Fernando Rodríguez-Gallego, Kassel, Reichenberger, 2015.

Calderón de la Barca, Pedro, Judas Macabeo, ed. Fernando Rodríguez-Gallego, Madrid / Frankfurt, Iberoamericana / Vervuert, 2012.

Calderón de la Barca, Pedro, La desdicha de la voz, autógrafo, BNE, Res. $108<$ http://www.cervantesvirtual.com/obra/la-desdicha-de-la-voz-manuscrito-comedia-inc-amiga-mia-ya-sabes-exp-que-perdoneis-nuestrasfaltas--0/> [04/07/2014]

Calderón de la Barca, Pedro, La devoción de la cruz, ed. Adrián Sáez, Madrid / Frankfurt, Iberoamericana / Vervuert, 2014.

Calderón de la BARCA, Pedro, La humildad coronada, autógrafo, BNE, Res. 72 <http://www.cervantesvirtual.com/obra-visor/la-humildad-coronada-manuscrito-auto-sacramental-para-la-ciudad-de-toledo-en-las-fiestasdel-santisimo-sacramento-ano-de-1644-inc-arboles-plantas-y-flores-explograr-la-corona-y-cenir-el-laurel--0/html> [04/07/2014].

Calderón de la BArCA, Pedro, La humildad coronada, ed. Ignacio Arellano, Pamplona / Kassel, Universidad de Navarra / Reichenberger, 2002. 
Calderón de la Barca, Pedro, La primera versión de "La vida es sueño» de Calderón, ed. José María Ruano de la Haza, Liverpool, Liverpool University Press, 1992.

Coenen, Erik, «El proceso de revisión en Calderón visto a través de sus comedias autógrafas», Anuario calderoniano, 8, 2015, pp. 71-92.

Ferrer Valls, Teresa (dir.), Diccionario biográfico de actores del teatro clásico español (DICAT), Kassel, Reichenberger, 2008 [DVD].

Greer, Margaret R., "Cazadores divinos, demoníacos y reales en los autos de Calderón de la Barca», en Divinas y humanas letras: doctrina y poesía en los autos sacramentales de Calderón, ed. Ignacio Arellano et al., Pamplona / Kassel, Universidad de Navarra / Reichenberger, 1997, pp. 217-244.

Kroll, Simon, «¿Cuántas versiones ofrece un autógrafo? Las intervenciones de autor en el autógrafo de El secreto a voces», Hipogrifo. Revista de literatura $y$ cultura del Siglo de Oro, 2.2, 2014, pp. 63-79.

Kroll, Simon, «Texto y actor: sobre dos repartos y una comedia de Calderón para la compañía de Antonio de Prado», en Nuevas sonoras aves. Catorce estudios sobre Pedro Calderón de la Barca, ed. Antonio Sánchez Jiménez, Madrid / Frankfurt, Iberoamericana / Vervuert, 2015, en prensa.

Mason, Robert A., «Introduction», en Pedro Calderón de la Barca, La desdicha de la voz, ed. Robert A. Mason, Liverpool, Liverpool University Press, 2003, pp. 1-116.

Rodríguez-Gallego, Fernando, "Aproximación a la reescritura de comedias de Calderón de la Barca», en Como en la antigua, en la edad nuestra. Presencia de la tradición en la literatura española del Siglo de Oro, ed. Natalia Fernández Rodríguez, Bellaterra, Prolope, 2010, pp. 157-193.

RuANo de la Haza, José María, «Las dos versiones de El mayor monstruo del mundo de Calderón», Criticón, 72, 1998, pp. 35-47.

Vitse, Marc, «Presentación», en Siglo de Oro y reescritura. I: Teatro, ed. Marc Vitse, Criticón, 72, 1998, pp. 5-10. 